\title{
A multiscale model for acid-mediated tumor invasion: therapy approaches
}

\author{
Gülnihal Meral ${ }^{1}$, Christian Stinner ${ }^{2}$, and Christina Surulescu2* \\ ${ }^{1}$ Bülent Ecevit University, Faculty of Arts and Sciences, \\ Department of Mathematics, 67100 Zonguldak, Turkey \\ ${ }^{2}$ Technische Universität Kaiserslautern, Felix-Klein-Zentrum für Mathematik, \\ Paul-Ehrlich-Str. 31, 67663 Kaiserslautern, Germany
}

(Dated: Friday $12^{\text {th }}$ September, 2014)

\begin{abstract}
Starting from the two-scale model for $\mathrm{pH}$-taxis of cancer cells introduced in [1], we consider here an extension accounting for tumor heterogeneity w.r.t. treatment sensitivity and a treatment approach including chemo- and radiotherapy. The effect of peritumoral region alkalinization on such therapeutic combination is investigated with the aid of numerical simulations.
\end{abstract}

Keywords: multiscale model, acid-mediated tumor invasion, $\mathrm{pH}$-taxis, radiotherapy, chemotherapy, reactiondiffusion-transport equations.

PACS: 87.10.Ed, 87.17.Jj, 87.18.Hf.

MSC: 92C17, 92C50, 35Q92, 35K10.

\section{INTRODUCTION}

Beside uncontroled proliferation of cells, tumor growth and cancer invasion are also characterized by alterations of the microenvironment in which they take place. The latter can be directly influenced by the cancer cells, e.g., by the proteins they secrete (ECM components, proteases, etc.) or can in turn have an impact on the dynamics of the neoplastic tissue, see e.g., [2]. A characteristic of invasive tumors is hypoxia, which results from an imbalance between oxygen supply and its consumption. Tumors have an extremely high energy demand for accomplishing the processes related to migration and proliferation and they satisfy this need by reinforced glycolysis, leading to acidification of their surroundings [3]. The adaptive advantages for metastatic progression conferred by dysregulated $\mathrm{pH}$ include degradation of normal tissue and alkalinization of the tumor cells' cytosol, with subsequent proliferation enhancement accompanied by polarization and directed migration, see e.g., [4].

Starting with the work of Gatenby \& Gawlinski [5], mathematical models have been proposed for the description of tumor cell invasion conditioned by acidosis. Most of them reconsider the model in [5] and possibly extend it, e.g., to account for vascular and avascular growth of multicellular tumor spheroids [6]. The settings involve reaction-diffusion equations for the densities of cancer cells and of normal cells, coupled with an equation for the concentration of extracellular protons, see also [7, 8]. All of these models, however, are set on the macroscopic scale of cell populations, whereas the microscopic, subcellular level is known

*Corresponding author. Email: surulescu@mathematik.uni-kl.de, Tel.: +49631205 5312, Fax: +49631 205-5308. to significantly influence (and even control) the macroscale behavior, e.g., by the intracellular proton dynamics, as mentioned above. Multiscale models offer a means for integrating detailed subcellular and individual information to allow predictions on the tumor level and they have also been used in a broader context, see e.g., [1, 9, 10] for two-scale models or [11-14] for settings involving several scales.

\section{MODEL}

The model introduced in [1] to characterize acid-mediated tumor invasion involved on the microscopic scale the intracellular proton dynamics. Thereby, the influence of proton extrusion by increase of acidity in the extracellular space was described by partial differential equations written on the macroscale for the densities of cancer cells and of normal cells, along with a reaction-diffusion equation for the concentration of extracellular protons, hence making the connection with the subcellular level. Cancer cells were found to bias their motion according to the $\mathrm{pH}$ gradient in their surroundings [15, 16]; the extracellular acidity is believed to boost the invasiveness and metastatic ability of cancer. This motivated in [1] the introduction of a corresponding taxis term in the equation for tumor cells. Having described in this way the spread of cancer, the next step would be to model therapy relying on the informations about tumor acidity and extent of invasion.

There exists, however, experimental evidence that the low extracellular $\mathrm{pH}\left(\mathrm{pH}_{e}\right)$ and the gradients between intracellular $\left(\mathrm{pH}_{i}\right)$ and $\mathrm{pH}_{e}$ significantly influence the response of tumors to various treatments like radiotherapy and chemotherapy [17- [22]. This suggests that a tumor is heterogeneous with respect to the treatment sensitivity of its constituent cells, depending on the local level of hypoxia: for instance, the latter is detrimental for radiotherapy, for 
which oxidation of the radiation-induced DNA free radicals is necessary for the subsequent apoptotic processes [23]. Also, in response to nutrient deprivation due to hypoxia, the rate of cancer cell proliferation decreases, but chemotherapeutic drugs are more effective against proliferating cells. We propose therefore to consider two subpopulations of cancer cells: those responding to treatment (we will denote with $a(t, \mathbf{x})$ their density function), and those exhibiting an enhanced resistance against therapy (represented in our model by the function $q(t, \mathbf{x})$ ). The latter are supposed to be the cells living in a low $p H_{e}$ environment, while those with a higher $p H_{e}$ are the more sensitive ones. Along with these two types of cancer cells our model also includes the evolution of normal cell density $n(t, \mathbf{x})$ and the dynamics of intracellular $y$ and extracellular $h(t, \mathbf{x})$ protons.

In the therapy strategy we propose here we follow a threefold aim: to reduce the peritumoral acidosis, to enhance the sensitivity of tumor cells to treatment, and to degrade the neoplastic tissue by chemo and radiotherapy, applied successively (the latter) or in a concurrent way (sensitivity enhancement by proton buffering and chemotherapy). The alkalinization could be achieved, e.g., by oral administration of bicarbonate [24] or trisodium citrate [25], which can increase the $\mathrm{pH}_{e}$ of tumors without notably influencing the blood or healthy tissue $\mathrm{pH}$. Systemic buffering is modelled below in (2) by a decay term in the equation for $h$, depending on the (time-varying) applied dosis $d_{c}$.

Many different ways have been proposed for the sensitization of tumor cells to therapy. Most chemotherapeuticals are weak acids or alkalines, hence they are neutralized in the acidic tumor microenvironment. However, this drawback can be overcome for instance by pretreatment with proton pump inhibitors, which reverts chemoresistance of several cancer cells to drugs like e.g., cisplatin, vinblastine, 5-fluorouracil, and doxorubicin and further increases the response of drug-sensitive cells to anticancer agents [20, 26]; other approaches using the same idea of increasing intracellular acidosis e.g., by inhibiting carbonic anhydrases were studied and/or reviewed in [27-30]. Moreover, intracellular acidification was shown to be cytotoxic to tumor cells, see e.g., [31], thus one can also achieve cell killing by applying such therapy. The influence of chemoterapeutic agents is modeled below by terms of the form $j \kappa_{j}\left(d_{c}\right) C_{j}\left(\theta_{j}, m\right)$, with $j \in\{a, q, n\}$, and depending on the cytotoxic drug concentration $m$. Thereby, the effect of treatment is expected to depend on the sensitivity of cancer cells, which in turn should be proportional to the dosis $d_{c}$ applied for the systemic buffering. Eventually, radiotherapy -described with terms of the form $j \Gamma_{j} R_{j}\left(\alpha_{j}, d_{r}\right)$, with $j \in\{a, q, n\}$ and $d_{r}$ denoting the time-dependent radiation dosis- will lead to enhanced depletion of cancer cells, favorized by the pre-treatment including alkalinization and cytotoxicity. As an example, we present here a treatment approach with buffering simultaneous to chemotherapy, followed by radiotherapy. If needed, alkalinization can also be applied before or only partially simultaneously to chemotherapy, before ir- radiation and after chemotherapy, etc.

With the coefficient functions (thereby $D_{q}, M, k_{1}, b, \nu, \omega_{1}$, $\omega_{2}, \omega_{3}$ and the carrying capacity $K_{n}$ of normal cells are positive constants and $C_{0}, H_{0}, Y_{0}$ denote the reference carrying capacity of cancer cells and the reference concentrations of extra- and intracellular protons, respectively)

$$
\begin{aligned}
& \varphi(a, q, n):=\frac{D_{q}}{1+\frac{(a+q) n}{C_{0} K_{n}}}, \quad f(a, q, h):=\frac{M q}{1+\frac{(a+q) h}{C_{0} H_{0}}}, \\
& \mu_{c}(y):=\frac{k_{1}}{1+\frac{y}{Y_{0}}}, \quad K_{c}(h):=C_{0}+b h, \\
& g(a+q):=\frac{\nu(a+q)}{1+\frac{a+q}{C_{0}}}, \quad \Theta(y, h):=\frac{\omega_{1} y}{1+\frac{y^{2}}{Y_{0}^{2}}+\omega_{2} h^{2}}-\frac{\omega_{3} h}{1+\frac{y^{2}}{Y_{0}^{2}}}
\end{aligned}
$$

our model then takes the following form:

$$
\left\{\begin{aligned}
\partial_{t} a= & \underbrace{\mu_{c}(y) a\left(1-\frac{a+q}{K_{c}(h(\cdot, t-\tau))}-\eta_{1} \frac{n}{K_{n}}\right)}_{\text {proliferation }} \\
& +\gamma\left(d_{c}\right) q-\lambda a-a \Gamma_{a} R_{a}\left(\alpha_{a}, d_{r}\right) \\
& -a \kappa_{a}\left(d_{c}\right) C_{a}\left(\theta_{a}, m\right), \\
\partial_{t} q= & \underbrace{\nabla \cdot(\varphi(a, q, n) \nabla q)}_{\text {nonlinear diffusion }} \underbrace{-\nabla \cdot(f(a, q, h) \nabla h)}_{\text {taxis }} \\
& +\lambda a-\gamma\left(d_{c}\right) q-q \Gamma_{q} R_{q}\left(\alpha_{q}, d_{r}\right) \\
& -q \kappa_{q}\left(d_{c}\right) C_{q}\left(\theta_{q}, m\right), \\
\partial_{t} n= & -\delta_{n} h n+\mu_{n} n\left(1-\eta_{2} \frac{a+q}{K_{c}(h(\cdot, t))}-\frac{n}{K_{n}}\right) \\
& -n \Gamma_{n} R_{n}\left(\alpha_{n}, d_{r}\right)-n \kappa_{n} C_{n}\left(\theta_{n}, m\right), \\
\partial_{t} h= & D_{h} \Delta h+\Theta(y, h)-\sigma_{c} d_{c} h \\
\partial_{t} y= & -\Theta(y, h)-\alpha y+g(a+q), \\
\partial_{t} m= & -\rho m+v_{m}(t),
\end{aligned}\right.
$$

where $a+q$ gives the total tumor cell density. All equations are set in $(0, T) \times \Omega$, where $\Omega \subset \mathbb{R}^{N}$ is a bounded domain with smooth boundary and $N \leq 3$. Concerning the spatial spread of cancer cells, they are assumed to diffuse and their motion to be biased by the $\mathrm{pH}$-gradient in their environment (following [15, 16] the latter was called in [1] pH-taxis). The nonlinear diffusion is influenced by the densities of tumor cells and of normal tissue, and is described by the diffusion coefficient $\varphi(a, q, n)$. The function $f(a, q, h)$ represents the $\mathrm{pH}$-tactic sensitivity of the moving cancer cells. Precise forms of $\varphi$ and $f$ are given in (1). In view of the "go-or-grow" dichotomy [32-34], we assume that only the non-proliferating cells are moving, and these are those which are less sensitive to therapy (hence in our notation the $q$ cells). For the tumor cell proliferation we consider logistic growth with crowding; the carrying capacity $K_{c}$ (a concrete form of which is given in (1)) is supposed to depend on the acidosis in the tumor microenvironment, hence we take it to be a function of the extracellular proton concentration $h$. The time delay expresses the fact that the adaptation of carrying capacity to the surrounding $\mathrm{pH}$ is not 
instantaneous. Relying on experimental evidence [35-37], we also consider a growth rate $\mu_{c}$ depending on the intracellular acidity, and choose it to cope with the observation that an alkaline cytosol is favorable to cell proliferation [38] (see (1) for a concrete form).

As we are primarily interested in the effects of acidosis on the tumor cells, the equation for the normal cells merely includes them in the terms involving the interaction with the cancerous tissue, i.e. in the degradation term modeling acidity induced apoptosis (with the constant decay rate $\delta_{n}$ ) and in the crowding term restricting proliferation. The extracellular proton buffering is supposed not to directly affect the normal tissue in a relevant way; its effects are rather indirect, by the nonlinear coupling of the equation for $n$ with the equations for the tumor cells and the extracellular protons. The function $\gamma\left(d_{c}\right)$ and the constant $\lambda \geq 0$ represent transition rates between $a$-cells and $q$-cells.

For the concrete choices of the functions modeling treatment we refer to (3) for radiotherapy and (4) for chemotherapy. The nonnegative coefficients $\Gamma_{j}$ and $\kappa_{j}$ are the corresponding death rates.

$$
R_{j}\left(\alpha_{j}, d_{r}\right)= \begin{cases}0, & \text { for } t \notin \text { radiotherapy } \\ 1-S\left(\alpha_{j}, d_{r}\right), & \text { for } t \in \text { radiotherapy }\end{cases}
$$

where $j \in\{a, q, n\}$ and "radiotherapy" denotes the set of times at which ionizing radiation is applied to the patient. Further, $S\left(\alpha_{j}, d_{r}\right)=\exp \left(-\alpha_{j} d_{r}-\beta_{j} d_{r}^{2}\right)$ models the survival fraction of each subpopulation $a, q$ or $n$ after the application of radiotherapy with a dosis $d_{r}$ (in Gy), hence we adopted the linear quadratic (LQ) model [39.41], which in spite of its shortcomings [42-44] is still the standard choice in radiation treatments. Thereby, $\alpha_{j}$ represents lethal lesions produced by a single radiation track (they are linearly related to the dose: $\alpha_{j} d_{r}$, cell kill per Gy), while $\beta_{j}$ characterizes lethal lesions produced by two radiation tracks (quadratically related to the dose: $\beta_{j} d_{r}$, cell kill per $\mathrm{Gy}^{2}$ ). The relevant parameter in the LQ model is actually the radiation sensitivity $\frac{\alpha_{j}}{\beta_{j}}$, which correlates to the cell cycle length: late responding tissues with a slow cell cycle have a small $\frac{\alpha_{j}}{\beta_{j}}$ ratio, while it is large for early responding, highly aggressive cancers [45]. In clinical practice the total dose $d_{r}$ is given in $N$ fractions of size $\hat{d}_{r}$, hence

$$
\begin{aligned}
S\left(\alpha_{j}, d_{r}\right) & =\exp \left(-N\left(\alpha_{j} \hat{d}_{r}+\beta_{j} \hat{d}_{r}^{2}\right)\right) \\
& =\exp \left(-\alpha_{j} d_{r}\left(1+\hat{d}_{r} /\left(\alpha_{j} / \beta_{j}\right)\right)\right) .
\end{aligned}
$$

For the chemotherapy description we consider the functions

$$
C_{j}\left(\theta_{j}, m\right)=\left\{\begin{array}{ll}
0, & \text { for } t \notin \text { chemotherapy } \\
1-\exp \left(-\theta_{j} m\right), & \text { for } t \in \text { chemotherapy }
\end{array}\right. \text {, }
$$

where analogously as before $j \in\{a, q, n\}$ and "chemotherapy" denotes the set of times at which a cytotoxic agent is administered. The chemotherapeutic effect depends on the concentration $m$ of the applied drug, while $\theta_{j}$ represents its depletion efficiency for the population $j$ of cells.

The proton dynamics involves the evolution of both intracellular protons of concentration $y$ and their extracellular counterparts with concentration $h$. As we are only interested in the spatiality w.r.t. the macroscale, only $h$ is diffusing. Due to the very small size of protons the diffusion coefficient can be assumed constant. In the absence of treatment and production by tumor cells, the reaction terms in the equations for $y$ and $h$ are due only to ion transport through the cell membrane. Thus, $\Theta(y, h)$ denotes the decay term for intracellular $\mathrm{H}^{+}$due to membrane transporters (e.g., NDCBE: $\mathrm{Na}^{+}$dependent $\mathrm{Cl}^{-}-\mathrm{HCO}_{3}^{-}$exchanger, NHE: $\mathrm{Na}^{+}$and $\mathrm{H}^{+}$exchanger, and AE: anion exchanger), production by aerobic glycolysis (possibly depending on microenvironmental vascularization), and intracellular buffering. The function $g(a+q)$ represents a source term due to the production (with saturation) by cancer cells. In order to maintain an advantageous intracellular $\mathrm{pH}$, cancer cells upregulate proton extrusion through membrane transporters, leading to acidosis of the tumor environment. The concentration of extracellular protons $\mathrm{H}^{+}$ is a macroscopic quantity explicitly depending on time and position. It is produced the same way the intracellular protons decay, by transport through the cell membrane. In our model it decays with a rate $\sigma_{c}$ due to systemic buffering induced by administration of bicarbonate with a dose $d_{c}$. We refer to (1) for concrete choices of these functions and to [9] for more details about the modeling of proton dynamics in 22. Finally, the evolution of the cytotoxic drug (we consider here doxorubicin) of concentration $m$ is influenced by the way it is made available at the tumor site $\left(v_{m}(t)\right.$ is the amount of doxorubicin injected per day per litre of body volume) and by the drug's decay with a rate $\rho \geq 0$. All constants involved in the model are nonnegative.

The equations (2) are supplemented with adequate initial and boundary conditions. For the latter we make the same choice as in [1] and assume no flux of cells or protons through the boundary of the domain of interest:

$$
\partial_{\mathbf{n}} q=\partial_{\mathbf{n}} h=0 \quad \text { on } \partial \Omega \times(0, T) .
$$

\section{GLOBAL EXISTENCE}

The local and global existence for (2) in the weak sense according to [1, Definition 3.1] is given in the following theorem.

Theorem 1 Let $\Omega \subset \mathbb{R}^{N}$ be a bounded domain with smooth boundary, $N \in\{1,2,3\}, \tau>0$, and assume that (1) is fulfilled. Furthermore, suppose that $\gamma\left(d_{c}\right), R_{j}\left(\alpha_{j}, d_{r}\right)$, $C_{j}\left(\theta_{j}, m\right), \kappa_{j}\left(d_{c}\right), d_{c}$, and $v_{m}$ are nonnegative functions of time belonging to $L^{\infty}((0, \infty))$ such that $\gamma\left(d_{c}(t)\right) \geq \gamma_{0}>0$ for all $t \geq 0$. If the initial data are nonnegative functions 
satisfying

$a_{0}, q_{0}, n_{0}, y_{0}, m_{0} \in C^{0}(\bar{\Omega}), h_{0} \in C^{0}\left([-\tau, 0] ; W^{1, q}(\Omega)\right)$, $n_{0} \leq K_{n}, y_{0} \leq Y_{0}$ in $\bar{\Omega}, \delta \leq h_{0} \leq H_{0}$ in $\bar{\Omega} \times[-\tau, 0]$

with some $q \in(N+2, \infty)$ and $\delta>0$, then there exists $T>0$ and $a$ weak solution to (2), (5) in $(0, T) \times \Omega$.

If in addition $c_{0} \in C^{\beta}(\bar{\Omega})$ holds with some $\beta \in\left(\frac{1}{N+2}, 1\right)$, then this weak solution is unique.

If we choose $\varphi(a, q, n):=\frac{D_{q}}{1+\frac{q n}{C_{0} K_{n}}}$, then a weak solution to (2), (5) exists globally in time.

The proof of this result parallels the one from [1, Theorem 3.2] and relies on deducing appropriate estimates and compactness properties for regularizations of (2). The main additional difficulty is the splitting of the cancer cells into the two subpopulations $a$ and $q$. As $a$ is proliferating and $q$ is migrating, the proof of estimates for $q$ in $L_{l o c}^{\infty}((0, \infty) \times \Omega)$ allowing to conclude the global existence (see [1, Lemmas 3.5 and 3.6]) is possible by suitable combinations of estimates involving $a, q$, and $a+q$, if the diffusivity $\varphi$ does not depend on $a$. A corresponding global result remains open for $\varphi$ from (1), in view of the lack of appropriate lower bounds for $\varphi$ in presence of the transition between $a$ and $q$.

\section{NUMERICAL RESULTS}

Before solving the model system (2) numerically, the nondimensionalization is made for the purpose of computational convenience. To this end, the dimensionless variables are taken as follows:

$$
\begin{aligned}
& \tilde{a}=\frac{a}{C_{0}}, \quad \tilde{q}=\frac{q}{C_{0}} \quad \tilde{n}=\frac{n}{K_{n}}, \quad \tilde{h}=\frac{h}{H_{0}}, \\
& \tilde{y}=\frac{y}{Y_{0}} \quad \tilde{x}=\frac{x}{L}, \quad \tilde{t}=\frac{t}{T}, \quad \phi=\frac{t}{\chi T}, \quad \tilde{m}=\theta_{a} m,
\end{aligned}
$$

where $L$ is the reference length scale and $T$ is the reference time unit, while $\chi$ and $\theta_{a}$ are some positive scaling constants.
The model in the nondimensionalized form becomes

$$
\left\{\begin{aligned}
\partial_{t} a= & \frac{k_{1}}{1+y} a\left(1-\frac{a+q}{K_{c}(h(\cdot, t-\tau))}-\eta_{1} n\right)-\lambda a \\
& +\left(\gamma_{0}+\gamma_{1} d_{c}\right) q-\Gamma_{a} a R_{a}\left(\alpha_{a}, d_{r}\right) \\
& -a\left(\kappa_{a 0}+\kappa_{a 1} d_{c}\right) C_{a}\left(\theta_{a}, m\right), \\
\partial_{t} q= & \nabla \cdot\left(\frac{D_{q}}{1+(a+q) n} \nabla q\right)-\nabla \cdot\left(\frac{M q}{1+(a+q) h} \nabla h\right) \\
& +\lambda a-\left(\gamma_{0}+\gamma_{1} d_{c}\right) q \\
& -\Gamma_{q} q R_{q}\left(\alpha_{q}, d_{r}\right) \\
& -q\left(\kappa_{q 0}+\kappa_{q 1} d_{c}\right) C_{q}\left(\theta_{q}, m\right), \\
\partial_{t} n= & -\delta_{n} h n+\mu_{n} n\left(1-\eta_{2} \frac{a+q}{K_{c}(h(\cdot, t))}-n\right) \\
& -\Gamma_{n} n R_{n}\left(\alpha_{n}, d_{r}\right)-n \kappa_{n} C_{n}\left(\theta_{n}, m\right), \\
\partial_{t} h= & D_{h} \Delta h+\frac{\gamma_{h} y}{1+y^{2}+\alpha_{h} h^{2}}-\frac{\beta_{h} h}{1+y^{2}}-\sigma_{c} d_{c} h, \\
\partial_{\phi} y= & -\frac{\gamma_{y} y}{1+y^{2}+\alpha_{h} h^{2}}+\frac{\beta_{y} h}{1+y^{2}}-\alpha y+\frac{\rho(a+q)}{1+(a+q)}, \\
\partial_{t} m= & -\rho m+\theta_{a} v_{m}(t)
\end{aligned}\right.
$$

The equations (6) are then supplemented with the initial conditions (Figure 1) and the boundary conditions (5). Here we assume that $70 \%$ of the cancer cells are less sensitive. In order to illustrate the qualitative behaviour we solve the mathematical model numerically using an explicit-implicit finite difference scheme as the one used in [10]. During the simulations we fixed the following parameters:

$$
\begin{aligned}
& \chi=0.01, D_{q}=2 * 10^{-4}, M=8 * 10^{-6}, k_{1}=10^{-1}, \\
& \mu_{n}=10^{-1}, \eta_{1}=0.35, \eta_{2}=0.05, \gamma_{0}=0.055, \\
& \lambda=0.076 .
\end{aligned}
$$

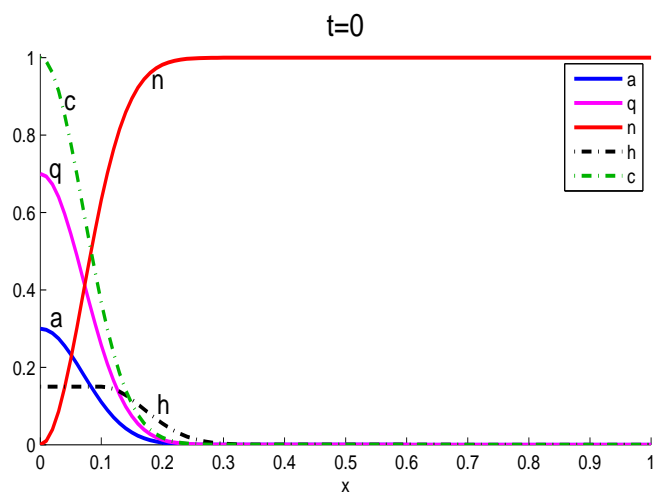

FIG. 1: Initial conditions. $c:=a+q$ denotes the total cancer cell density.

The treatment schedule considered here involves three weeks of buffering combined with chemotherapy followed by seven weeks of radiotherapy (with no irradiation during weekends) [47]. For the chemotherapy we use the recommended dosage for doxorubicin and assume that the amount $(\mathrm{mg})$ injected per day per litre of body $\left(v_{m}\right)$ is 2.3869 [46]. 

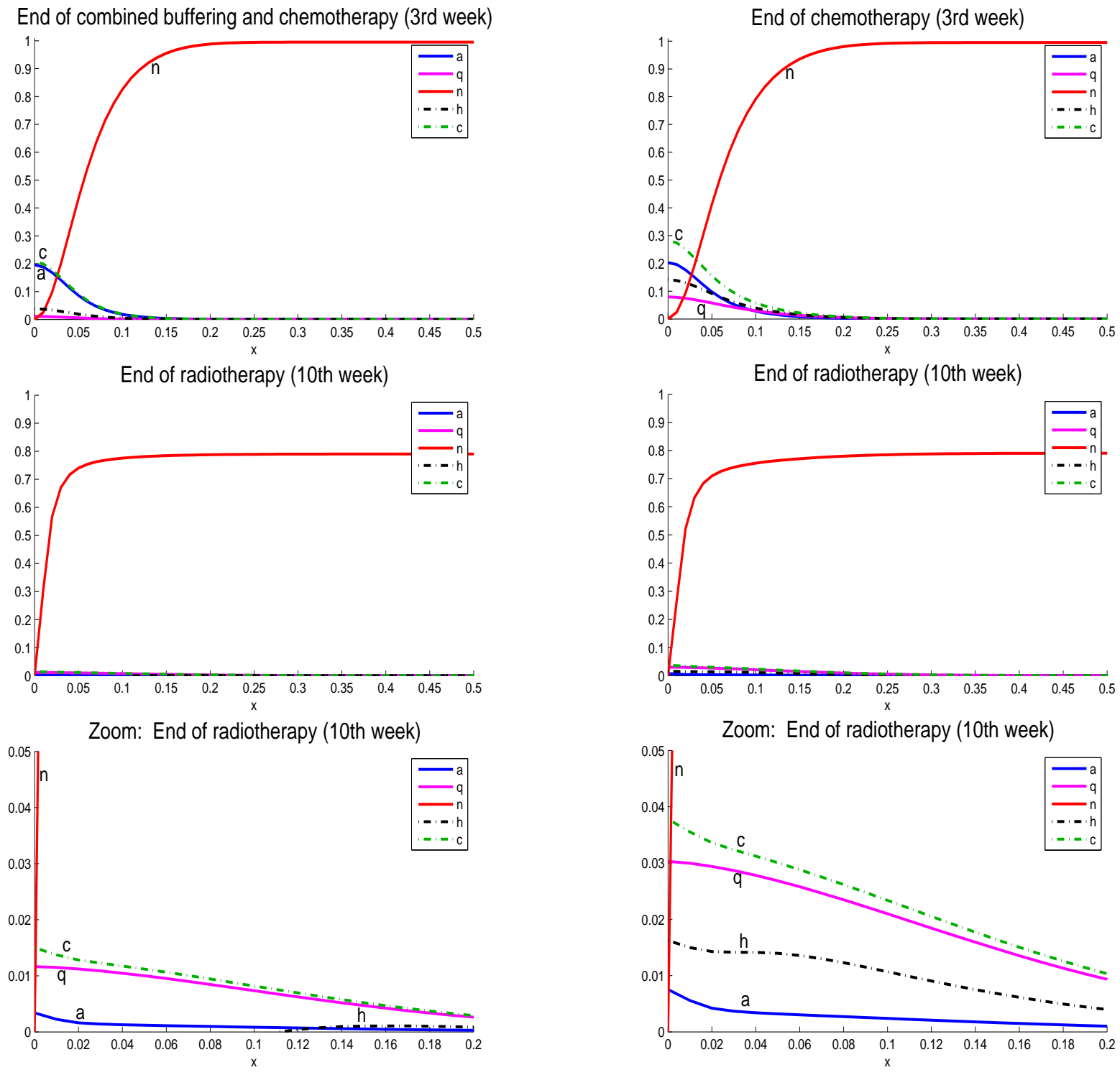

FIG. 2: Evolution of cancer cells, normal cells and exterior protons at different times for combined buffering and chemotherapy ( 3 weeks) followed by radiotherapy (7 weeks) tons at different times for chemotherapy ( 3 weeks) followed by radiotherapy (7 weeks), but without buffering
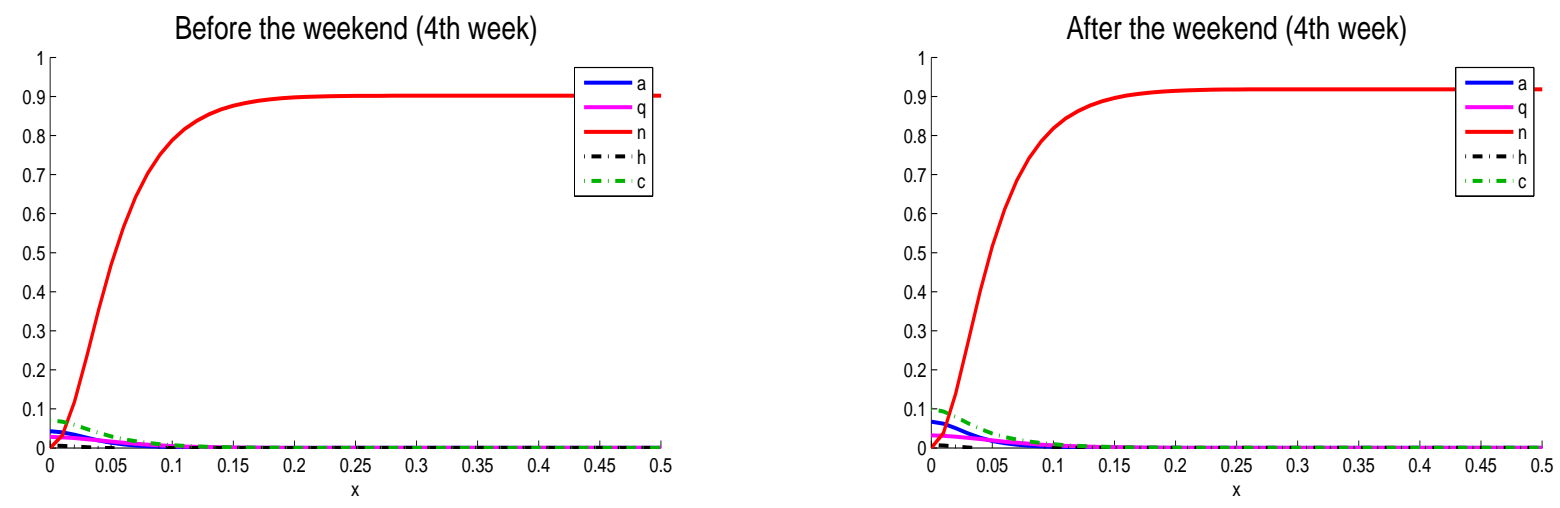

FIG. 3: Before the weekend of the 4th week (setting of FIG.2

FIG. 5: After the weekend of the 4th week (setting of FIG.2] 
The initial drug concentration is assumed to be zero. Following [46], the drug efficacy coefficients for both types of cancer cells are taken to be 1.8328 , whereas for the normal cells we choose a 10 times smaller value, since the drug is supposed to be less effective for normal cells. For the radiotherapy we fixed the ratio $\alpha_{a} / \beta_{a}=10$, corresponding to a head and neck cancer [39] and considered smaller fixed ratios for the less sensitive cancer cells $\left(\alpha_{q} / \beta_{q}=0.7\right)$ and for the normal cells $\left(\alpha_{n} / \beta_{n}=0.1\right)$. We also used in the simulations a total dosis not exceeding $61,2 G y+5 \%$, according to the standard treatment protocol [47].

Figure 2 illustrates the case with systemic buffering, while Figure 4 shows the case with only chemo and radiotherapy, without cell sensitization via proton reduction. The former strategy leads to an enhanced therapeutical outcome. The effect of weekend breaks during the radiotherapy is exemplified in Figures 3 and 5 . This pausing in the radiationinduced cell killing allows the normal, but also the tumor cells to recover (to a certain extent) and usually leads to a prolongement of the overall treatment time. This behavior is also confirmed by our model.

\section{DISCUSSION}

We considered in this paper a multiscale setting for acidmediated tumor invasion relying on the model in [1]. The present model supplementary involves treatment, thereby explicitly accounting for tumor heterogeneity w.r.t. the sensitivity of cancer cells to therapy. Such selective behavior is due to a plethora of causes, however here it is considered to be inflicted by the local acidity in the tumor microenvironment [17-21]. Reducing acidosis in the peritumoral region has been found not only to enhance the tumor's response to both chemo and radiotherapy, see, e.g., [19, 25, 26, 48], but also to alleviate invasiveness [3, 6, 24]. With our model accounting for two different types (according to their sensi- tivity) of cancer cells interacting with the normal tissue and conditioned by the dynamics of intra- and extracellular protons, we propose a mathematical framework for verifying these assertions. Reducing extracellular acidosis increases the sensitivity of tumor cells, thus enhancing the ratio of sensitive, hence proliferating (and rather non-motile) neoplastic tissue in detriment of the less sensitive (but more mobile) cancer cells. The transition rate between the two tumor cell types then obviously depends on the dosis of the proton buffering substance (e.g., bicarbonate) and so does the therapy efficiency. The numerical simulations confirm the biological observations: the therapeutic impact is augmented through extracellular proton buffering and the invasion is implicitly reduced.

There are, of course, many different therapy combinations possible, including successive and/or concurrent ones. The best choice would represent an interesting, however quite complicated optimum problem, which is even more difficult to solve as long as no reliable datasets are available and most parameters still have to be taken from different literature sources. The model presented here offers one way of describing the micro-macro dynamics involved in the highly complex process of cancer invasion and depletion by some therapeutical approach. It can be further reduced, while still preserving its two-scale features, e.g., by renouncing some of the parameters or simplifying the diffusion and/or taxis coefficients, according to which particular issue of the problem is being investigated.

\section{Acknowledgments}

Gülnihal Meral acknowledges the support by the German Academic Exchange Service (DAAD) during her visit in July 2014 to the University of Kaiserslautern. Ch. Surulescu acknowledges the support of the German Research Foundation DFG.

\section{References}

[1] C. Stinner, C. Surulescu, and G. Meral, A multiscale model for $\mathrm{pH}$-tactic invasion with time-varying carrying capacities, preprint TU Kaiserslautern, (2014).

[2] D. Hanahan and R.A. Weinberg, Cell, 144, 646, (2011).

[3] C. Stock and A. Schwab, Eur. J. Physiology, 458, 981, (2009).

[4] C. Frantz, A. Caridis, P. Nalbant, K.M. Hahn, and D.L. Barber, J. Cell Biol. 179, 403, (2007).

[5] R.A. Gatenby and E.T. Gawlinski, Cancer Res., 56, 5745, (1996).

[6] K. Smallbone, D.J. Gavaghan, R.A. Gatenby, and P.K. Maini, J. of Theor. Biol., 235, 476, (2005).

[7] A. Fasano, M. Herrero, and M.R. Rodrigo, Math. Biosci., 220, 45, (2009).
[8] C. Maerkl, G. Meral, and C. Surulescu, Int. J. of Analysis, 2013, 15, (2013).

[9] S. Hiremath and C. Surulescu, A stochastic multiscale model for acid mediated cancer invasion, Nonl. Anal. Real World Appl., to appear.

[10] G. Meral, C. Stinner, and C. Surulescu, On a Multiscale Model Involving Cell Contractivity and its Effects on Tumor Invasion, Discrete Cont. Dyn. Syst. B, to appear.

[11] N. Bellomo, A. Bellouquid, J. Nieto, and J. Soler, Math. Models Methods Appl. Sci., 22, 37pp, (2012).

[12] J. Kelkel and C. Surulescu, Math. Models Methods Appl. Sci., 23, (2012).

[13] T. Lorenz and C. Surulescu, Math. Models Methods Appl. Sci., 24, 54pp, (2014).

[14] C. Engwer, T. Hillen, M.P. Knappitsch, and C. Surulescu, Glioma Follow White Matter Tracts; a Multiscale DTI-based Model, J. Math. Biol., to appear. 
[15] P. Bartel, F.T. Ludwig, A. Schwab, and C. Stock, Acta Physiologica, 204, 113, (2012).

[16] R.K. Paradise, M.J. Whitfield, D.A. Lauffenburger, and K.J. Van Vliet, Experimental Cell Research, 319, 487, (2013).

[17] M.L. Freeman and E. Sierra, Radiat Resist, 97, 154, (1984).

[18] H.-S. Lee, H.J. Park, J.C. Lyons, R.J. Griffin, E.A. Auger, and C.W. Song, Int. J. Radiat. Oncol. Biol. Phys. , 38, 1079, (1997).

[19] C.W. Song, R. Griffin, and H.J. Park, in Cancer Drug Resistance. Edited B. Teicher, Humana Press Inc., Totowa, NJ (2006).

[20] A. De Milito and S. Fais, Future Oncol., 1, 779, (2005).

[21] N. Raghunand, X. He, R. van Sluis, B. Mahoney, B. Baggett, C.W. Taylor, G. Paine-Murrieta, D. Roe, Z.M. Bhujwalla, and R.J. Gillies, Br. J. Cancer, 80, 1005, (1999).

[22] G.L. Semenza, Cancer Cell, 5, 405, (2004).

[23] P. Ebbesen, E.O. Pettersen, T.A. Gorr, G. Jobst, K. Williams, J. Kieninger, R.H. Wenger, S. Pastorekova, L. Dubois, P. Lambin, B.G. Wouters, T. Van den Beucken, C.T. Supuran, L. Poellinger, P. Ratcliffe, A. Kanopka, A. Görlach, M. Gasmann, A.L. Harris, P. Maxwell, and A. Scozzafava, J. Enzyme Inhib. Med. Chem., 24, 1, (2009).

[24] A.S. Silva, J.A. Yunes, R.J. Gillies, and R.A. Gatenby, Cancer Res., 69, 2677, (2009).

[25] B. Requena, M. Zabala, P. Padial, and B. Feriche, J. Strength Cond. Res., 19, 213, (2005).

[26] F. Luciani, M. Spada, A. De Milito, A. Molinari, L. Rivoltini, A. Montinaro, M. Marra, L. Lugini, M. Logozzi, F. Lozupone, C. Federici, E. Iessi, G. Parmiani, G. Arancia, F. Belardelli, and S. Fais, J Natl Cancer Inst, 96, 1702, (2004).

[27] J. Doyen, S.K. Parks, S. Marcié, J. Pouysségur, and J. Chiche, Front. Oncol., 2, 1, (2013).

[28] C.T. Supuran, Bioorg. Med. Chem. Lett., 20, 3467, (2010).

[29] S. Pastorekova, S. Parkkila, and C.T. Supuran, J. Enzyme Inhib. Med. Chem., 19, 199, (2004).

[30] A. Maresca, C. Temperini, L. Pochet, B. Masereel, A. Scozzafava, and C.T. Supuran, J. Med. Chem. 53, 335, (2010).

[31] C.W. Song, H.J. Park, and B.D. Ross, in Drug Resistance in Oncology. Edited B.D. Teicher, Humana Press, Totowa, (1998).

[32] A. Giese, M.A. Loo, N. Tran, D. Haskett, S.W. Coons, and M.E. Berens, Int. J. Cancer, 67, 275, (1996).

[33] K.S. Hoek, O.M. Eichhoff, N.C. Schlegel, U. Döbbeling, N. Kobert, L. Schaerer, S. Hemmi, and R. Dummer, Cancer Res., 68, 650, (2008).

[34] P.P. Zheng, L.A. Severijnen, M. van der Weiden, R. Willemsen, and J.M. Kros, Cell Cycle, 8, 950, (2009).

[35] J. Chiche, C.M. Brahimi-Horn, and J. Pouysségur, J. of Cellular and Molecular Medicine, 14, 771, (2010).

[36] S. Harguindey, G. Orive, J. Luis Pedraz, A. Paradiso, and S.J. Reshkin, Biochim Biophys Acta - Reviews on Cancer, 1756, 1, (2005).

[37] S. Wu, T. Song, S. Zhou, Y. Liu, G. Chen, N. Huang, and L. Liu, Biochem. Biophys. Res. Comm., 375, 384, (2008).

[38] R.J. Gillies, R. Martinez-Zaguilan, E.P. Peterson, and R. Perona, Cell Physiol. Biochem., 2, 159, (1992).

[39] J. Fowler, Br. J Radiol, 62, 679, (1989).

[40] E. Hall and A. Giaccia, Radiobiology for the radiologist, Lipincott Williams \& Wilkins, (2006).

[41] R.K. Sachs, D.J. Brenner, Med. Phys., 25, 2071, (1998)

[42] D.J. Brenner, L.R. Hlatky, P.J. Hahnfeldt, E.J. Hall, and R.K. Sachs, Int. J. Rad. Onc. Biol. Phys., 32, 379, (1995).

[43] J.P. Kirkpatrick, J.J. Meyer, and L.B. Marks, Semin. Rad. Oncol., 18, 240, (2008).

[44] B.G. Woulters and J.M. Brown, Radiat. Res., 147, 541, (1997).

[45] G.G. Steel (ed.), Basic Clinical Radiobiology, 3rd ed., Arnold, (2002).

[46] L. Pillis, K. R. Fister, W. Gu, C. Collins, M. Daub, D. Gross, J. Moore, and B. Preskill, Comput. Math. Method M, 10(3), 165, (2009).

[47] R. Rockne, E.C. Alvord Jr., J.K. Rockhill, and K.R. Swanson, Math. Bio., 58, 561, (2009).

[48] M.F. McCarty and J. Whitaker, Altern. Med. Rev., 15, 264, (2010). 